\title{
Caspase-3 as an important factor in the early cytotoxic effect of nickel on oral mucosa cells in patients treated orthodontically
}

\author{
Piotr Buczko ${ }^{1}$, Izabela Szarmach ${ }^{1}$, Monika Grycz ${ }^{1}$, Irena Kasacka ${ }^{2}$ \\ ${ }^{1}$ Department of Orthodontics, Medical University of Bialystok, Bialystok, Poland \\ ${ }^{2}$ Department of Histology and Cytophysiology, Medical University of Bialystok, Bialystok, Poland
}

\begin{abstract}
Introduction. The effect of fixed orthodontic appliances on biochemical changes in saliva and pathophysiological status of the oral cavity is not clear. Recent data showed that nickel (Ni) released from orthodontic appliances can decrease cellular viability, induce DNA damage and apoptosis in oral mucosa cells. Since the mechanism of these Ni effects is unknown, the aim of our study was to analyze the expression of caspase-3 in epithelial cells of oral mucosa in healthy individuals treated orthodontically.

Material and methods. Twenty-eight volunteers participated in the study. Epithelial cells were collected from oral mucosa directly before appliance insertion, one week after the insertion, and 24 weeks after the insertion of fixed appliances. Cellular identification and measurements were conducted by light microscopy. Caspase-3 expression was evaluated immunohistochemically. Nickel concentration in saliva was also determined.

Results. A significantly higher number of oral epithelial cells with caspase-3 immunoreactivity was found one week, but not 24 weeks, after orthodontic treatment. The enhanced expression of caspase-3 was accompanied by increased nickel concentration in saliva.

Conclusions. Our data suggests that nickel released from orthodontic appliances can activate caspase-3 and this mechanism may be partially responsible for the cytotoxic action of nickel in the oral cavity of orthodontically-treated individuals. (Folia Histochemica et Cytobiologica 2017, Vol. 55, No. 1, 37-42)
\end{abstract}

Key words: orthodontic appliances; epithelial oral mucosa cells; caspase-3; nickel; saliva; IHC

\section{Introduction}

The use of orthodontic appliances in the treatment of various maxillary dental abnormalities creates a very complex environment in oral cavity. Nickel chromium alloys are widely used in dentistry for the construction of prosthetic devices such as crowns, bridges and metal frames. Such alloys are mainly composed of $68-80 \%$ nickel and $12-26 \%$ chromium but they also contain other elements in quantities varying from

Correspondence address: P. Buczko, M.D., Ph.D

Department of Orthodontics,

Medical University of Bialystok

Waszyngtona St. 15 A, 15-269 Bialystok, Poland

tel: +48606272403

e-mail: pbuczko@op.pl
0.1 to $14 \%$ [1,2]. Recent studies indicate an increased release of nickel from orthodontic appliances into the artificial saliva or into human salivary secretions [3-5]. Moreover, a few studies have demonstrated a positive correlation between the enhanced nickel concentration in oral mucosa cells released from fixed orthodontic appliances and DNA damage as well as increased number of apoptotic cells $[6,7]$.

There is a limited number of studies investigating the mechanisms through which metals, in general, and nickel, in particular, determine apoptosis $[1,6,7]$. Within this context the relationship between apoptosis of oral epithelial cells and nickel content of orthodontic devices is unknown.

Apoptosis typically occurs through one of two signaling cascades known as extrinsic and intrinsic 
pathways, both of which converge to activate the so-called executioner caspase-3 [8]. In $2012 \mathrm{Yu}$ et al. observed an inhibitory effect of nickel on the proliferation of human gingival fibroblasts due to its potential of inducing apoptosis via caspase-3 activation [9]. This observation suggests nickel involvement in the apoptotic action of caspase- 3 in the oral cavity of individuals treated orthodontically. Indeed, in the study by Shi et al. nickel-free stainless steel appliances did not change the expression of caspase-3 in the culture of L929 cells in comparison with Co-Cr alloys and $\mathrm{Au}$ alloys [10].

To our knowledge, no results of in vivo studies on the role of caspase- 3 in the toxicity of nickel released from orthodontic appliances have been published. Therefore, the aim of our study was to investigate the effect of orthodontic treatment on the morphology of oral mucosa epithelial cells and caspase-3 immunoreactivity immediately before and one, and 24 weeks after nickel-containing appliance insertion in healthy individuals.

\section{Material and methods}

Patients treatment and material sampling. Oral epithelial cells were collected from 28 dentistry students aged 21-24 from the Medical University of Bialystok, Poland. Out of the 28 participants, 5 displayed a Class II malocclusion, 4 had crossbite and all subjects had dental crowding. The participants were fully informed of the nature of the study and written consent was obtained. The Ethics Committee of the Medical University of Bialystok (permission number R-I-002/67/2012) approved the study.

All participants were clinically stable non-smokers, and they did not report any health problems. The subjects had not taken medication which could impact saliva composition 6 months prior to the commencement of the study and were not suffering from any systemic diseases and chronic or acute oral infections (mucositis or candidiasis). The participants were instructed and screened once a week in all aspects of oral hygiene by a qualified dentist in the month preceding the attachment of the fixed appliances. Following the insertion of 3M Victory Series brackets (3M Unitek Orthodontic Products, Monrovia, CA, USA), the participants received instructions concerning the need for supplementary oral hygiene measures (orthodontic toothbrushes, dental floss, mouth rinsing). All appliances were inserted by two dentists. 3M Victory Series steel brackets contain approximately $3 \%$ nickel and $15 \%$ chromium, $3 \%$ cuprum and less than $1 \%$ of other elements. Epithelial cells were collected by scrape cytology on three occasions, immediately before appliance insertion, one week after the treatment and 24 weeks after the attachment of the fixed appliances. Smears of the epithelial cells were immediately fixed with $96 \%$ ethanol.
Two cytological swabs and smears were obtained from each individual. Study participants were instructed to refrain from food for two hours before cell collection, which was performed between 8 and 9 a.m.

Cytological and immunohistochemical staining and evaluation. The smears were stained using the May-Grünwald-Giemsa method and processed by immunohistochemistry for caspase-3. The EnVision method was used to reveal antibody-antigen reactions according to Herman and Elfont [11].

The immunostaining was performed according to the following protocol. For antigen retrieval, the slides with ethanol-fixed smears of oral epithelial cells were pretreated with a Target Retrieval Solution (S 1699; Dako, Glostrup, Denmark) in a pressure chamber at $125^{\circ} \mathrm{C}$ and 21 psi for $1 \mathrm{~min}$. After being cooled to room temperature, smears were incubated with Peroxidase Blocking Reagent (S 2001; Dako) for $10 \mathrm{~min}$ to block endogenous peroxidase activity. Slides were incubated overnight at $4^{\circ} \mathrm{C}$ in a humidified chamber with the diluted CASPASE 3 Rabbit anti-Human Polyclonal Antibody (1:1,000, LS-B3404; LifeSpan Biosciences, Inc., Seattle, WA, USA) followed by the incubation with a secondary antibody (Kit EnVision (+) K 4011; Dako) conjugated to horseradish peroxidase-labeled polymer. Bound antibodies were visualized by a 1 min incubation with liquid 3,3'-diaminobenzidine substrate chromogen (DAB; Dako). Finally, the smears were counterstained with QS hematoxylin (H-3404; Vector Laboratories Inc., Burlingame, CA, USA), mounted and evaluated by light microscopy. Appropriate washings with Wash Buffer (S 3006; Dako) were performed between each step. The specificity tests performed for the CASPASE 3 antibody included negative control, where the antibodies were replaced by normal rabbit serum (Vector Laboratories) at the required dilution, and positive control, using human spleen as a specific tissue recommended by the manufacturer.

Identification and measurements of cells were conducted using Olympus BX41 light microscope (Olympus, Tokyo, Japan), set at total $200 \times$ magnification and examined by an independent observer in a blind fashion. Oral epithelial cells were examined with regard to their shape, size and the nuclear-to-cellular area ratio. After taking photographs with a digital camera (DP12-2; Olympus), the cellular area (CA), nuclear area (NA) and nuclear-to-cellular area (NA-CA) ratio were measured using a computerized image analysis program, NIS Elements BR: 2240 (Nikon, Tokyo, Japan). Fifty cells from each slide were examined morphometrically. The mean values of CA, NA and NA-CA ratio were obtained for each patient.

The presence of caspase- 3 in the epithelial cells of oral mucosa was assessed according to the number of immunoreactive (brown) cells. Fifty cells were analyzed in each smear, and values were given as a number of caspase-3-positive 
cells/50 cells. The intensity of the immunohistochemical (IHC) reaction was measured using a 0 to 256 grey scale level where a completely black pixel received the value of 0 , whereas the one with the value of 256 was completely white or bright.

Determination of nickel concentration in saliva. Nickel concentration was measured according to Kocaderelli et al., as previously described, in each sample of unstimulated saliva $[12,13]$. Briefly, mixed saliva $(3 \mathrm{~mL})$ samples were checked for food, blood or nasal discharge contamination and contaminated samples were excluded. Then, $3 \mathrm{~mL}$ of saliva was transferred into a beaker and $20 \mathrm{~mL}$ of $2 \%$ nitric acid $\left(\mathrm{HNO}_{3}\right)$ was added. Samples were filtered into a volumetric flask using Whatman No. 42 filter paper. The filtrates were then diluted to the final volume of $100 \mathrm{~mL}$ with distilled water and then stored at $-20^{\circ} \mathrm{C}$ until analyzed. The saliva samples were analyzed for nickel content with an atomic absorption spectrophotometer with a graphite oven (Solar M, ThermoElectron Corp., Madison, WI, USA). Nickel concentration levels were calculated as micrograms per milliliter.

Statistical analysis. The results are presented as mean \pm standard deviation (SD). The data were statistically analyzed by means of the software computer package Statistica ver. 12.0 (Satsoft Corp., Tulsa, OK, USA). The significance of differences was determined by one-way ANOVA test, and the level of statistical significance was set at $\mathrm{p}<0.05$ level.

\section{Results}

Immediately before insertion of the appliances epithelial cells of the oral mucosa displayed similar shapes and size (Table 1, Fig. 1A). One week after the insertion of the appliances numerous changes in the morphology of oral mucosa epithelial cells were found (Fig. 1B). Cells' shape and size became less regular since a number of significantly enlarged cells as well as a number of substantially smaller cells were observed. Additionally, cell nuclei of various size were detected in one field of vision. Some cells had large, vesicular nuclei while in others the nuclei were very small, pyknotic and darkly-stained, which resulted in the significant decrease of the NA-CA ratio as compared to the pre-insertion values (Table 1). Twenty-four weeks after the procedure, morphological alterations in the epithelial cells were much less pronounced than the ones previously observed in the subjects before orthodontic treatment. Some cells exhibited only slight changes in shape and size (Fig. 1C). However, morphometric measurements revealed that the NA-CA ratio was comparable to that observed one week after procedure (Table 1).

The immunohistochemical detection of caspase- 3 revealed a positive reaction in the cytoplasm of oral mucosal epithelial cells in all study groups (Fig. 2A, B and $\mathrm{C}$ ). There were significant differences in the number of caspase-3-immunoreactive cells at different time periods following the attachment of fixed orthodontic appliances (Table 1). Only a few caspase-3-positive cells/50 negative cells were found in the smears of subjects before orthodontic appliance insertion. We observed a greater than two-fold increase in the number of caspase-3-positive oral epithelial cells in the smears of subjects one week after appliance insertion as compared to pre-insertion values (Fig. 2A, B and Table 1). Twenty-four weeks after the procedure the number of caspase-3-immunoreactive cells was comparable to that observed prior to the procedure (Fig. 2A, C and Table 1).

The intensity of caspase- 3 immunostaining was the most pronounced, according to a 0 to 256 grey scale level, in the smears of subjects one week after appliance insertion, while it was similar 24 weeks after orthodontic treatment when compared to the pre-insertion value (Table 1).

A significant increase in nickel concentration in saliva one week after orthodontic treatment (24.1 $\pm 4.2 \mathrm{ng} / \mathrm{mL} ; \mathrm{p} \geq 0.001)$ as compared to the values obtained before orthodontic treatment $(0.2 \pm 0.01 \mathrm{ng} / \mathrm{mL})$ and 24 weeks thereafter $(2.2 \pm 0.3 \mathrm{ng} / \mathrm{mL})$.

Table 1. Morphometry of oral mucosa epithelial cells and number of caspase-3-immunoreactive cells after orthodontic treatment

\begin{tabular}{|l|c|c|c|c|c|}
\hline $\begin{array}{l}\text { Time after ortho- } \\
\text { dontic appliance } \\
\text { insertion }\end{array}$ & $\begin{array}{c}\text { Cytoplasmic area } \\
{\left[\mu \mathbf{m}^{2}\right]}\end{array}$ & $\begin{array}{c}\text { Nuclear area } \\
{\left[\mu \mathbf{m}^{2}\right]}\end{array}$ & $\begin{array}{c}\text { Nuclear-cytopla- } \\
\text { smic ratio }\end{array}$ & $\begin{array}{c}\text { Number of caspase-3 } \\
\text { Ir-cells/50 cells in } \\
\text { a viewfield }\end{array}$ & $\begin{array}{c}\text { Intensity of IHC } \\
\text { reactivity }\end{array}$ \\
\hline 0 weeks & $1816.77 \pm 933.27$ & $76.36 \pm 28.56$ & $0.0463 \pm 0.015$ & $8.2 \pm 0.43$ & $149.9 \pm 2.21$ \\
\hline One week & $1823.04 \pm 799.86$ & $57.89 \pm 21.98^{*}$ & $0.0361 \pm 0.017^{*}$ & $19.5 \pm 2.13^{*}$ & $126.9 \pm 4.60^{*}$ \\
\hline 24 weeks & $22687.17 \pm 617.10^{*}$ & $80.48 \pm 26.21$ & $0.0367 \pm 0.011^{*}$ & $11.6 \pm 1.25$ & $147.8 \pm 3.38$ \\
\hline
\end{tabular}

Data present mean $\pm \mathrm{SD}, \mathrm{n}=28$. For morphometric measurements cells were stained by the May-Grunewald-Giemsa method and the immunohistochemical detection of caspase-3 was performed as described in Methods. Ir, immunoreactivity. * significantly different from the values before treatment, $\mathrm{p} \geq 0.05$. 

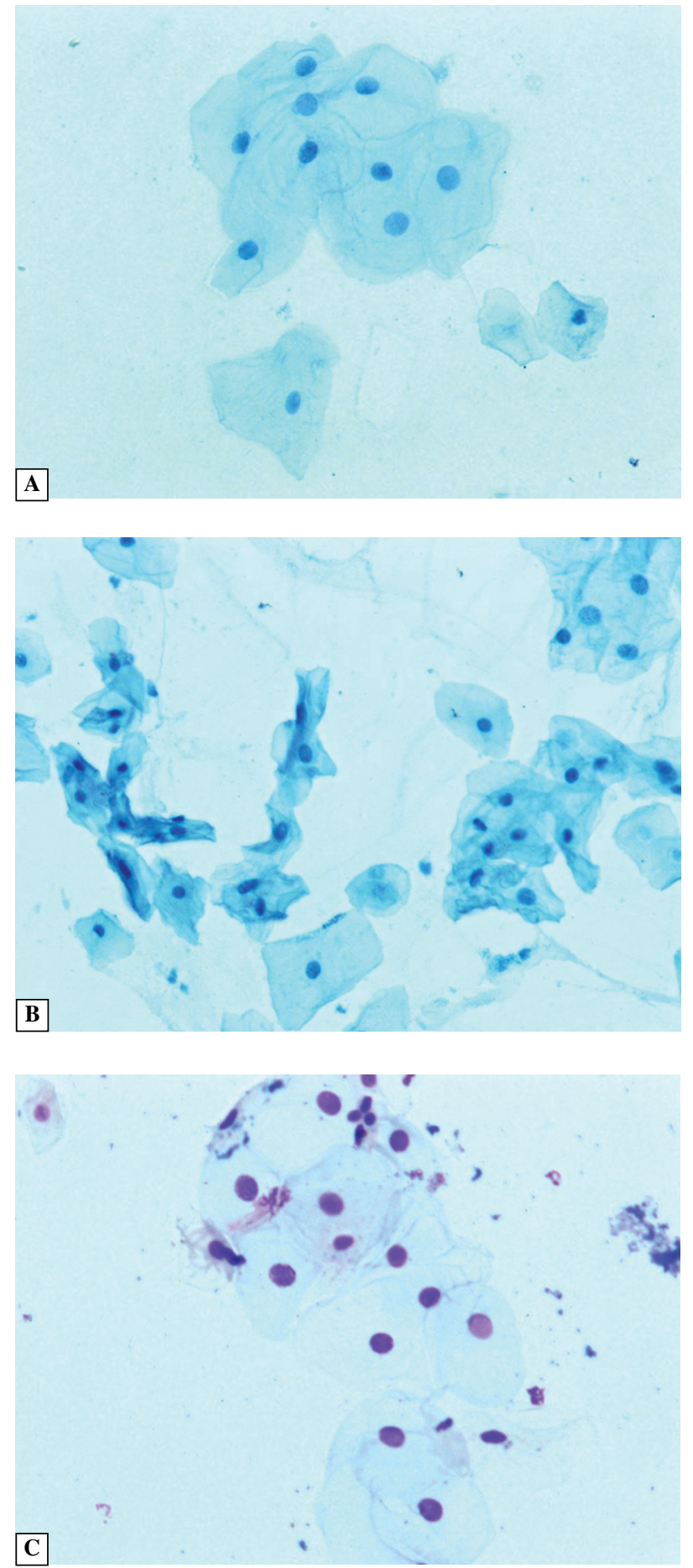

Figure 1. The morphology of oral mucosa epithelial cells. The smears of patients: before (A), one week (B) and 24 weeks (C) after orthodontic treatments. Smears were stained by the May-Grünwald-Giemsa method. Magnification $\times 200$.

\section{Discussion}

We investigated the morphological picture of oral epithelium cells in orthodontic patients wearing fixed appliances. We also evaluated the immunoreactivity of
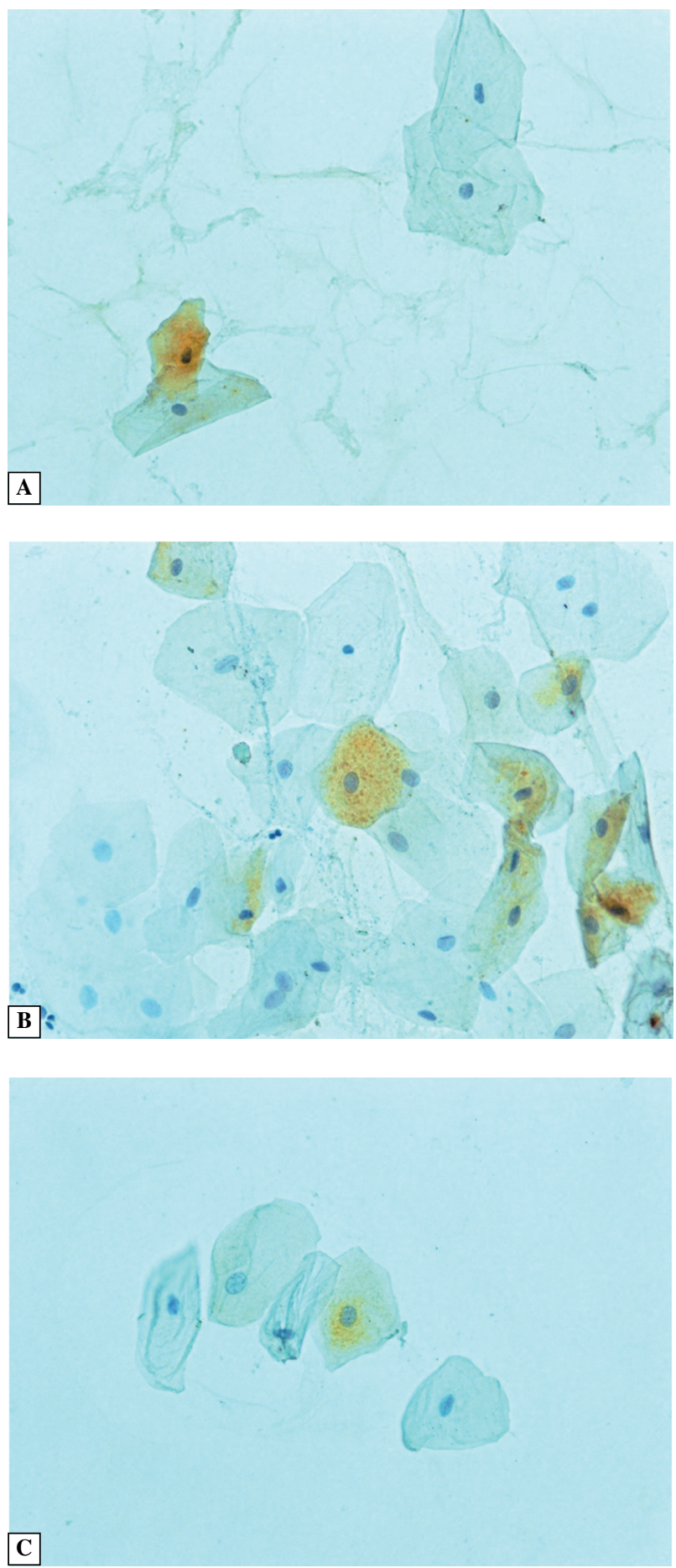

Figure 2. The immunohistochemical detection of caspase- 3 in the oral mucosa epithelial cells. The smears of patients: before (A), one week (B) and 24 weeks (C) after orthodontic treatments. Smears were immunostained with polyclonal antibody that specifically detects the cells with activated caspase-3. Magnification $\times 200$.

apoptosis-related protein caspase-3 in the buccal epithelial cells and nickel, a potentially toxic element contained in orthodontic appliances, concentration in saliva. 
We observed numerous changes in the morphology of oral mucosa cells in the smears of subjects one week after appliance insertion. These changes were accompanied by an enhanced number of cells with an enhanced expression of caspase-3 in oral epithelial cells and the and augmented nickel levels in the subjects' saliva.

During the last decade, a limited number of papers indicating that orthodontic treatment can be a factor inducing cytotoxicity of mucosa cells in the oral cavity has been published. It has been well established that orthodontic appliances release nickel into the artificial saliva and into the saliva of oral cavity $[1,6,7,14]$. Indeed, Faccioni et al. found a higher nickel concentration in the oral mucosa cells of patients treated orthodontically in comparison to controls [6]. A greater number of comet and apoptotic cells were also observed in patients with a higher saliva nickel concentration suggesting that the metal released from orthodontic appliances could induce DNA damage in oral epithelial cells. However, the results of various studies are not consistent. Hafez et al. demonstrated that fixed orthodontic appliances decreased cell viability and induced DNA damage [7] and the toxic changes were correlated with the cellular nickel content of the oral mucosa cells. Nataranjan et al. observed a significantly higher micronucleus frequency in the group treated with fixed orthodontic appliances but the differences were not statistically significant 30 days after deboning. Additionally, at that time no significant differences were found in nickel content between the tested and control groups. The authors concluded that orthodontic appliances released nickel in quantities sufficient to induce a localized genotoxic effect only during the orthodontic treatment [15]. These data are supported by in vitro studies. Soesetijo et al. have recently demonstrated enhanced apoptosis of gingival fibroblasts induced by nickel released from nickel-chromium alloys [1]. Latvala et al. found that nickel can enhance reactive oxygen species generation, cytotoxicity and genotoxicity in human alveolar epithelial cell line A549 [14]. Our present and previous results [13] are in concordance with the aforementioned data. We also found that in clinically healthy individuals, orthodontic appliances released nickel ions into the saliva one week, but not 24 weeks, after orthodontic treatment. Moreover, the simultaneous augmentation of the markers of total oxidative stress in the saliva was also found [13]. Our present morphological study demonstrated a decrease in the nuclear area and nuclear-cytoplasmic ratio in oral mucosa cells one week after orthodontic treatment. By contrast, the cytoplasmic area of mucosa cells
24 weeks after treatment was statistically higher in respect to the pre-treatment value. These changes seem to be dependent on the chronic mechanical irritation of the oral cavity by orthodontic appliances and/or by enhanced apoptosis induced by the longterm release of nickel. As a consequence, at least through this mechanism the hypertrophy of mucosa cells, as reflected by decreased nuclear-cytoplasmic ratio after one week of treatment, could have been induced.

It has been shown that oxidative stress, which induces apoptosis, depends on the activation of caspase-3 in human lens epithelial cells, rat dopaminergic neuronal N27 cells and mice testicular cells [16-18]. Our immunohistochemical staining revealed a higher number of caspase-3 immunoreactive epithelial cells in clinically healthy participants one week after the attachment of orthodontic appliances. It has been well established that caspase-3 is necessary for apoptotic chromatin condensation and DNA fragmentation [19]. Furthermore, it has been demonstrated that the activation of caspase- 3 is associated with oxidative stress [20,21]. Soesetijo et al. have recently demonstrated high apoptosis of gingival fibroblasts treated with nickel-containing artificial saliva [1]. These data support our hypothesis that an orthodontic appliance releases nickel which induces cell damage by enhancing oxidative stress and via caspase- 3 activation augments apoptosis in epithelial oral mucosa cells [13]. To our knowledge, this is the first in vivo study indicating the involvement of caspase signaling pathways in the mechanism of toxicity caused by nickel released from fixed orthodontic appliances in clinically healthy individuals. Lack of toxic effects 24 weeks after orthodontic treatment may indicate tolerance for nickel or removal of the metal from buccal epithelial cells as suggested by Hafez et al. [7]. The majority of previous studies were performed in vitro or on small numbers of participants, mainly children, from different countries, with different diets and different oral hygiene standards. Since oral hygiene is crucial for the clinical status of the oral cavity, our present study was performed on clinically healthy individuals with no detectable pathological changes in the oral cavity. Although in vivo studies are useful in explaining how orthodontic materials interact with oral mucosa cells, the interpretation of the obtained results is usually difficult due to a number of factors which may affect the results, however, cannot be under full experimental control. Therefore, despite careful observation of the healthy volunteers who participated in the study, some other, difficult-to-define factors other than increased nickel saliva levels, might have influenced the obtained results. 
In conclusion, our data indicate that after one week of treatment with orthodontic appliances increased saliva nickel levels are associated with caspase- 3 activation and probably via this mechanism nickel can induce apoptosis in the oral mucosa epithelial cells of healthy individuals treated orthodontically.

\section{Acknowledgment}

The study was supported by the Medical University of Bialystok, Poland, grant No. 144-07503L.

\section{Conflict of interest}

The authors report no conflict of interest.

\section{References}

1. Soesetijo FXA, Darmanto W, Prijatmoko D, et al. The effect of nickel as product of recasting nickel chromium on gingival fibroblasts apoptosis. J Int Dent Med Res. 2015; 8(1): 25-30.

2. Craig RG, Power JM. Restorative Dental Materials, 11th ed. Mosby Inc, St Louis Missouri 2002.

3. Gölz L, Knickenberg AC, Keilig L, et al. Nickel ion concentrations in the saliva of patients treated with self-ligating fixed appliances: a prospective cohort study. J Orofac Orthop. 2016; 77(2): 85-93, doi: 10.1007/s00056-016-0012-x, indexed in Pubmed: 26910844.

4. Hussain HD, Ajith SD, Goel P. Nickel release from stainless steel and nickel titanium archwires - An in vitro study. J Oral Biol Craniofac Res. 2016; 6(3): 213-218, doi: 10.1016/j.jobcr.2016.06.001, indexed in Pubmed: 27761386.

5. Fariborz A, Alireza J, Parviz A, et al. Metal ion release from fixed orthodontic appliances an in vivo study. Eur J Orthod. 2012; 34(1): 126-130, doi: 10.1093/ejo/cjq181, indexed in Pubmed: 21303810

6. Faccioni F, Franceschetti P, Cerpelloni M, et al. In vivo study on metal release from fixed orthodontic appliances and DNA damage in oral mucosa cells. Am J Orthod Dentofacial Orthop. 2003; 124(6): 687-93; discussion 693, doi: 10.1016/S0889540603007418, indexed in Pubmed: 14666083.

7. Hafez HS, Selim EM, Kamel Eid FH, et al. Cytotoxicity, genotoxicity, and metal release in patients with fixed orthodontic appliances: a longitudinal in-vivo study. Am J Orthod Dentofacial Orthop. 2011; 140(3): 298-308, doi: 10.1016/j. ajodo.2010.05.025, indexed in Pubmed: 21889074.

8. Almeida A. Genetic determinants of neuronal vulnerability to apoptosis. Cell Mol Life Sci. 2013; 70(1): 71-88, doi: 10.1007/s00018-012-1029-y, indexed in Pubmed: 22695677.

9. Yu J, Zhao F, Wen X, et al. Apoptosis mechanism of gingival fibroblasts induced by nickel ion contained in dental cast alloys. Biomed Mater Eng. 2012; 22(1-3): 151-157, doi: 10.3233/BME-2012-0701, indexed in Pubmed: 22766714.

10. Shi T, Qin T, Zhan D. Evaluation of biocompatibility of the medical nickel-free stainless steel by detecting expression of caspase-3. Oral Biomed. 2010; 03: 010.

11. Herman GE, Elfont EA. The taming of immunohistochemistry: the new era of quality control. Biotech Histochem. 1991; 66(4): 194-199, indexed in Pubmed: 1912080.

12. Kocadereli L, Ataç PA, Kale PS, et al. Salivary nickel and chromium in patients with fixed orthodontic appliances. Angle Orthod. 2000; 70(6): 431-434, doi: 10.1043/0003-3219(2000)070 $<0431$ :SNACIP > 2.0.CO;2, indexed in Pubmed: 11138646.

13. Buczko P, Knaś M, Szarmach I, et al. The impact of orthodontic treatment on antioxidant capacity of saliva. Adv Med Sci. 2016.

14. Latvala S, Hedberg J, Di Bucchianico S, et al. Nickel Release, ROS Generation and Toxicity of Ni and NiO Micro- and Nanoparticles. PLoS One. 2016; 11(7): e0159684, doi: 10.1371/journal.pone.0159684, indexed in Pubmed: 27434640.

15. Natarajan M, Padmanabhan S, Chitharanjan A, et al. Evaluation of the genotoxic effects of fixed appliances on oral mucosal cells and the relationship to nickel and chromium concentrations: an in-vivo study. Am J Orthod Dentofacial Orthop. 2011; 140(3): 383-388, doi: 10.1016/j.ajodo.2010.07.027, indexed in Pubmed: 21889083.

16. Yao H, Tang X, Shao X, et al. Parthenolide protects human lens epithelial cells from oxidative stress-induced apoptosis via inhibition of activation of caspase- 3 and caspase-9. Cell Res. 2007; 17(6): 565-571, doi: 10.1038/cr.2007.6, indexed in Pubmed: 17339884.

17. Carvour M, Song C, Kaul S, et al. Chronic low-dose oxidative stress induces caspase-3-dependent PKCdelta proteolytic activation and apoptosis in a cell culture model of dopaminergic neurodegeneration. Ann N Y Acad Sci. 2008; 1139: 197-205, doi: 10.1196/annals.1432.020, indexed in Pubmed: 18991865.

18. Kalia S, Bansal MP. Regulation of apoptosis by Caspases under oxidative stress conditions in mice testicular cells: in vitro molecular mechanism. Mol Cell Biochem. 2009; 322(1-2): 43-52, doi: 10.1007/s11010-008-9938-7, indexed in Pubmed: 18979186.

19. Savitskaya MA, Onishchenko GE, Savitskaya MA, et al. Mechanisms of Apoptosis. Biochemistry (Mosc). 2015; 80(11): 1393-1405, doi: 10.1134/S0006297915110012, indexed in Pubmed: 26615431.

20. Labbé D, Teranishi MA, Hess A, et al. Activation of caspase-3 is associated with oxidative stress in the hydropic guinea pig cochlea. Hear Res. 2005; 202(1-2): 21-27, doi: 10.1016/j. heares.2004.10.002, indexed in Pubmed: 15811695.

21. Tan SN, Sim SP, Khoo ASB. Potential role of oxidative stress-induced apoptosis in mediating chromosomal rearrangements in nasopharyngeal carcinoma. Cell Biosci. 2016; 6: 35, doi: 10.1186/s13578-016-0103-9, indexed in Pubmed: 27231526. 